\title{
Frontières
}

\section{Quelques réflexions sur le travail en équipe et les soins palliatifs}

\section{Suzanne Mongeau et Claudette Foucault}

Volume 18, numéro 2, printemps 2006

La mort dans tous ses états

URI : https://id.erudit.org/iderudit/1073222ar

DOI : https://doi.org/10.7202/1073222ar

Aller au sommaire du numéro

Éditeur(s)

Université du Québec à Montréal

ISSN

1180-3479 (imprimé)

1916-0976 (numérique)

Découvrir la revue

Citer cet article

Mongeau, S. \& Foucault, C. (2006). Quelques réflexions sur le travail en équipe et les soins palliatifs. Frontières, 18(2), 51-56. https://doi.org/10.7202/1073222ar
Résumé de l'article

En soins palliatifs, le travail en équipe est nécessaire, pertinent mais difficile. Cet article précise les difficultés qui y sont reliées. Les caractéristiques des équipes qualifiées de plus ou moins vivantes et celles des équipes qualifiées de plus ou moins mortifères sont ensuite dégagées. Des repères pour faciliter l'aménagement des difficultés du travail en équipe sont présentés. d'utilisation que vous pouvez consulter en ligne.

https://apropos.erudit.org/fr/usagers/politique-dutilisation/ 


\section{Résumé}

En soins palliatifs, le travail en équipe est nécessaire, pertinent mais difficile. Cet article précise les difficultés qui y sont reliées. Les caractéristiques des équipes qualifiées de plus ou moins vivantes et celles des équipes qualifiées de plus ou moins mortifères sont ensuite dégagées. Des repères pour faciliter l'aménagement des difficultés du travail en équipe sont présentés.

Mots clés: travail en équipe soins palliatifs - interdisciplinarité difficultés.

\section{Abstract}

In palliative care, teamwork is necessary and pertinent, yet complex. This article underlines the difficulties related to it. Characteristics of more or less functional and dysfunctional teams are then presented. Indications aiming to resolve teamwork difficulties are then proposed.

Keywords: teamwork - palliative care interdisciplinary - difficulties.

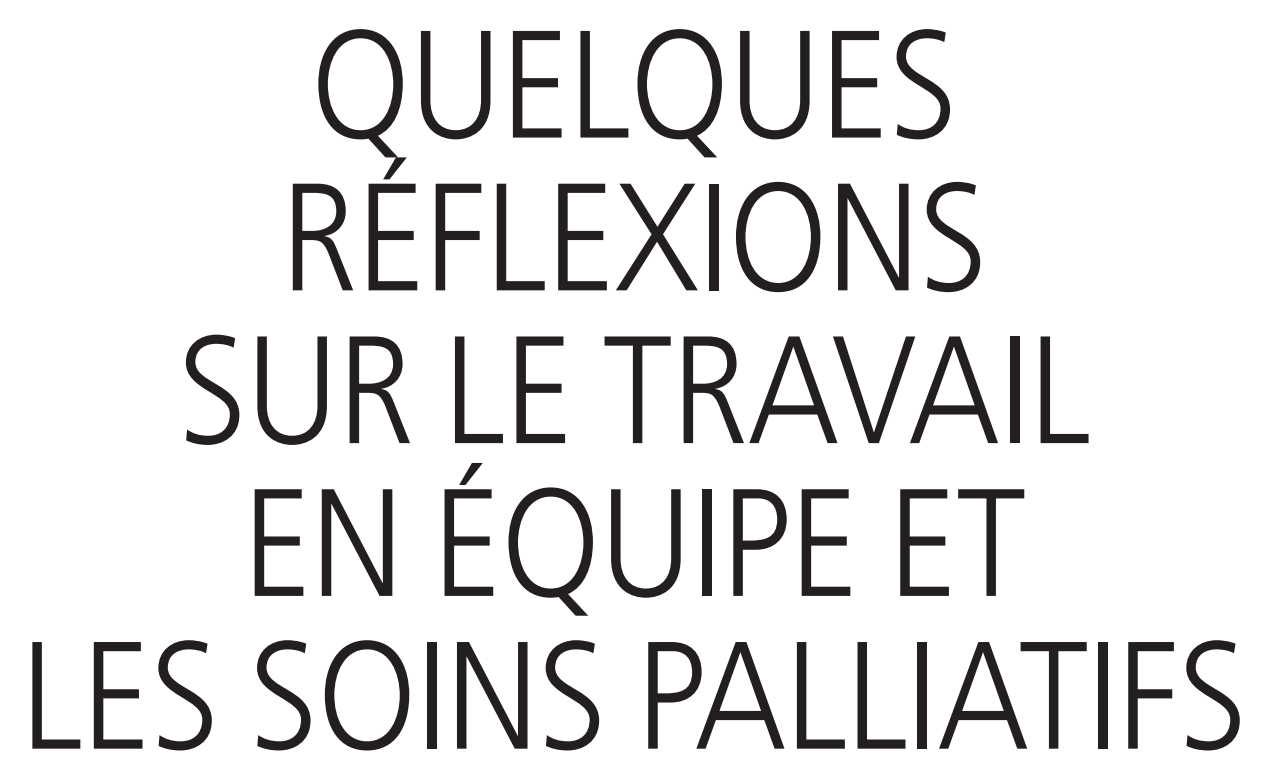

Suzanne Mongeau, Ph.D., professeure, École de travail social, UQAM.

\section{Claudette Foucault, \\ conseillère clinique en soins palliatifs, CSSS de Laval.}

En soins palliatifs, on reconnaît sans ambivalence la pertinence et même plus, la nécessité de travailler en équipe. En fait, la philosophie même des soins palliatifs le commande. Les textes fondateurs de cette philosophie mentionnent que les soins palliatifs reposent sur la prise en compte de la personne malade dans toutes ses dimensions: physique, psychologique, sociale et spirituelle (Lamau, 1994). Différents écrits présentent l'équipe interdisciplinaire non seulement comme la meilleure façon d'actualiser la philosophie même des soins palliatifs mais aussi, comme le souligne Saunders (1990), comme une façon de soutenir et récompenser ceux qui travaillent dans ce domaine exigeant. Or, comme nous l'indiquons dans la présente contribution, il semble qu'il ne soit pas toujours évident que l'équipe remplisse sa mission de soutien et de compensation.

Plusieurs études qui se sont penchées sur les stress vécus dans le travail en soins palliatifs en sont arrivées à la conclusion suivante: la source de stress la plus importante n'est pas le patient mourant et sa famille, mais il semble que le plus grand stress soit plutôt relié au travail en équipe et à l'organisation des soins (Vachon, 1987; Barnes, 2001; Musumeci, 2001; Lafontaine, 2001; Fillion et al., 2003). À la suite de son étude menée auprès de soignants œuvrant en soins palliatifs, Mary Vachon (1987) en est même arrivée à affirmer qu'en ce qui concerne les situations de stress vécues dans les milieux de soins palliatifs, le patient mourant ne constitue pas le véritable problème.

Ces résultats ne signifient pas pour autant que la confrontation avec la mort, ainsi que certains dilemmes éthiques rencontrés en fin de vie, ne sont pas des facteurs de stress. On peut en effet se demander si l'immersion constante au cœur d'expériences de séparation, de perte, de grande souffrance, donc dans des registres d'intensité émotive élevée, ne peut pas amener certains membres des équipes à adopter une posture de plus en plus rigide.

Le travail d'équipe en soins palliatifs est nécessaire, pertinent, mais difficile. Nous nous appliquerons ici à identifier les difficultés qui y sont reliées et à dégager les caractéristiques des équipes qualifiées de plus ou moins vivantes et celles des équipes qualifiées de plus ou moins morti- 
fères. Nous proposerons ensuite des repères pour faciliter l'aménagement des difficultés du travail en équipe. Pour élaborer le contenu de cette contribution, nous avons utilisé trois sources de données. Nous avons consulté les écrits de plusieurs auteurs sur le travail d'équipe et les soins palliatifs, ainsi que dans le plus vaste domaine du travail en équipe et de l'interdisciplinarité. Nous avons aussi fait appel à des informateurs clés. Au cours des dernières années, nous avons interrogé informellement plusieurs personnes œuvrant dans le domaine des soins palliatifs. Enfin, nous nous référons à notre expérience personnelle dans les diverses équipes de travail où nous avons œuvré en soins palliatifs.

\section{DIFFICULTÉS RENCONTRÉES DANS LES UNITÉS ET LES MAISONS DE SOINS PALLIATIFS}

Pour aborder les principales difficultés rencontrées dans le travail d'équipe en soins palliatifs, nous les regroupons en deux grandes catégories. Dans un premier temps, nous présenterons les difficultés rencontrées dans les unités et les maisons de soins palliatifs. Celles qui sont vécues dans les hôpitaux n'ayant pas d'unité de soins palliatifs et dans les CLSC seront présentées et analysées par la suite.

L'une des difficultés rencontrées est reliée à l'exercice même de l'interdisciplinarité. Il convient tout d'abord de rappeler quelques définitions, puisque dans ce domaine on confond souvent les notions de monodisciplinarité, de multidisciplinarité et d'interdisciplinarité (Dussault, 1986). La monodisciplinarité suppose que les intervenants travaillent en parallèle, voire en compétition. La multidisciplinarité, quant à elle, implique qu'il y ait juxtaposition des différentes expertises. L'exercice de la multidisciplinarité dans une réunion d'équipe, par exemple, fait en sorte que chaque professionnel expose son point de vue en se référant à sa discipline de spécialisation. Les interactions se font donc au niveau de la référence et de la consultation. En interdisciplinarité, il y a intégration des différentes expertises. On parle alors d'un effet de synergie puisque dans l'interdisciplinarité le bénéfice obtenu devrait être plus grand que celui résultant de l'addition des diverses contributions individuelles des professions. On suppose que quelque chose de plus devrait être créé à partir de ce regard intégrateur. L'exercice de l'interdisciplinarité demande que les interactions se fassent dans une optique de coordination de l'action, de collaboration interprofessionnelle et de partage des tâches. Qu'est-ce qui pose plus spécifiquement problème dans l'exercice de l'interdisciplinarité? On constate que les différents intervenants et soignants ont été très mal ou peu préparés à travailler en interdisciplinarité. Nous avons tous été formés dans une logique de professionnalisation (D'Amour et al., 1999) et cette logique est en bonne partie centrée sur la défense des territoires professionnels. Dans une telle perspective, les frontières entre les différentes professions doivent demeurer le plus étanches possible. De plus, on aspire à ce que chaque profession développe son propre langage ainsi que son propre cadre de référence. Or l'exercice de l'interdisciplinarité, comme nous l'avons mentionné au préalable, suppose un partage des diverses zones d'intervention. Dans l'exercice de l'interdisciplinarité, on doit asseoir à une même table une infirmière, un médecin, un aumônier, une travailleuse sociale, une pharmacienne et leur demander de considérer ensemble ce dont le patient et sa famille ont véritablement besoin, et non pas ce que les diverses professions ont besoin de donner à un patient mourant et à sa famille. Cette forme de sociabilité caractéristique de l'interdisciplinarité n'est pas toujours évidente si on a été formé selon une logique de professionnalisation. La pratique de l'interdisciplinarité offre à chacun des membres la possibilité de donner son point de vue, et ce, en sachant que donner son opinion ne veut pas nécessairement dire se rendre au chevet du malade ou rencontrer sa famille. Cette pratique n'est pas non plus évidente si on est quelqu'un qui a un grand besoin d'aider ou de jouer un rôle de sauveur, ce que l'on retrouve fréquemment en soins palliatifs. Ce sont probablement ces mêmes constatations qui ont conduit Lassaunière et Plagès (1995) à dire: «Nous sommes [...] des handicapés de l'interdisciplinarité» (p. 38).

Certaines attitudes peuvent favoriser le bon fonctionnement de l'interdisciplinarité. Hébert $(1990,1997)$ soutient qu'un sentiment de sécurité personnelle et professionnelle est un préalable essentiel pour établir les limites de sa compétence et éviter que le travail d'équipe soit un mécanisme de compensation à une compétence mal intégrée ou mal assumée. Dans l'exercice de l'interdisciplinarité, il faut arriver à prendre sa place au sein d'un territoire à partager. L'ouverture à l'inconnu constitue un autre élément aidant. Le travail en interdisciplinarité requiert une attitude positive à l'égard des nouvelles avenues. Après la lecture du livre de Guy Bourgeault intitulé Éloge de l'incertitude (1999), nous ajoutons à cette liste la capacité de douter. Travailler au sein d'une équipe interdisciplinaire demande de tolérer d'être ébranlé et remis en question. Bien souvent, on devra fonctionner dans l'incertitude. Il s'agira de partager nos questions plutôt que nos réponses. La valorisation des différences, une autre attitude favorisant l'interdisciplinarité, permet de considérer les autres professionnels avec moins de préjugés. Selon Hébert (1990, 1997), l'acceptation d'une certaine interdépendance constitue également une autre attitude favorable. Il s'agit en fait de reconnaître qu'on a besoin des autres, qu'il est important de ne pas penser et agir seul. Or il n'est pas si simple d'adopter une telle attitude car il y aurait chez l'aidant un désir latent d'agir seul avec l'aidé. Il y a souvent un certain plaisir, une satisfaction, à être le principal aidant. De plus, comme le disait une intervenante chevronnée, on retrouve en soins palliatifs beaucoup de «sauveurs» et de «bon monde» qui ont un grand besoin d'aider. Or tout partage peut diminuer la satisfaction $\mathrm{du}$ "grand sauveur». Enfin, toujours selon Hébert, une attitude systémique est nécessaire à l'exercice de l'interdisciplinarité, puisqu'elle permet d'aborder l'intervention ou la situation en se donnant une vue d'ensemble.

Une autre difficulté mentionnée concerne les rôles des différents membres d'une équipe en soins palliatifs. On observe un chevauchement des rôles qui crée des rivalités et des luttes de pouvoir, entrâ̂nant une certaine ambiguïté (Cummings, 1998; Fillion et al., 2003; Vachon, 1987). Il semble également qu'en soins palliatifs on ait tendance à éviter de clarifier les attentes au niveau des rôles. Ce chevauchement nous apparaît toutefois inévitable puisqu'en soins palliatifs, une grande place est accordée à l'accompagnement. Rappelons ici que l'accompagnement n'est pas réservé à une discipline en particulier. Cette difficulté quant au rôle constitue un nœud important. On observe également une certaine tendance à dévaloriser le rôle dit instrumental, comme celui de donner des injections, remplir des formulaires, etc. En soins palliatifs, on accorde en effet une très grande importance à l'aspect relationnel et la dimension spirituelle. Il semble que cela ait contribué à ce que les aspects plus instrumentaux des rôles occupent une place moindre. Pourtant, en soins palliatifs, on est impliqué auprès de gens gravement malades «dans leur corps» et auprès de proches devenus soignants souvent malgré eux qui, dans le présent contexte, ont besoin de recevoir des services à domicile, ce qui exige entre autres que des formulaires soient remplis.

L'aménagement des conflits constitue une autre difficulté. Les équipes de soins palliatifs font face à des difficultés bien spécifiques lorsqu'il s'agit de composer avec les conflits. Nos informateurs clés, de même que certains auteurs, signalent que les équipes de soins palliatifs ont tendance à adopter des stratégies d'évitement (Vachon, 1987 ; Musumeci, 2001). Plusieurs éléments 
peuvent expliquer ce phénomène. Lévesque (1992) observe qu'à notre époque, nous avons tendance à avoir une perception négative des conflits. Les conflits sont perçus comme indésirables, préjudiciables, voire dangereux, alors que dans les faits, ils sont inévitables dans les rapports humains. En fait, les écrits sur la médiation préconisent même la nécessité du conflit comme un élément de la qualité de vie d'une organisation. Dans cette perspective, les conflits peuvent amener des changements intéressants puisqu'ils nous obligent à revoir nos règles, nos rôles, nos croyances, notre fonctionnement. Un autre facteur pouvant expliquer qu'on ait tendance à adopter des stratégies d'évitement lorsqu'on fait face aux conflits a trait à l'histoire même des soins palliatifs. Les gens impliqués en soins palliatifs sont

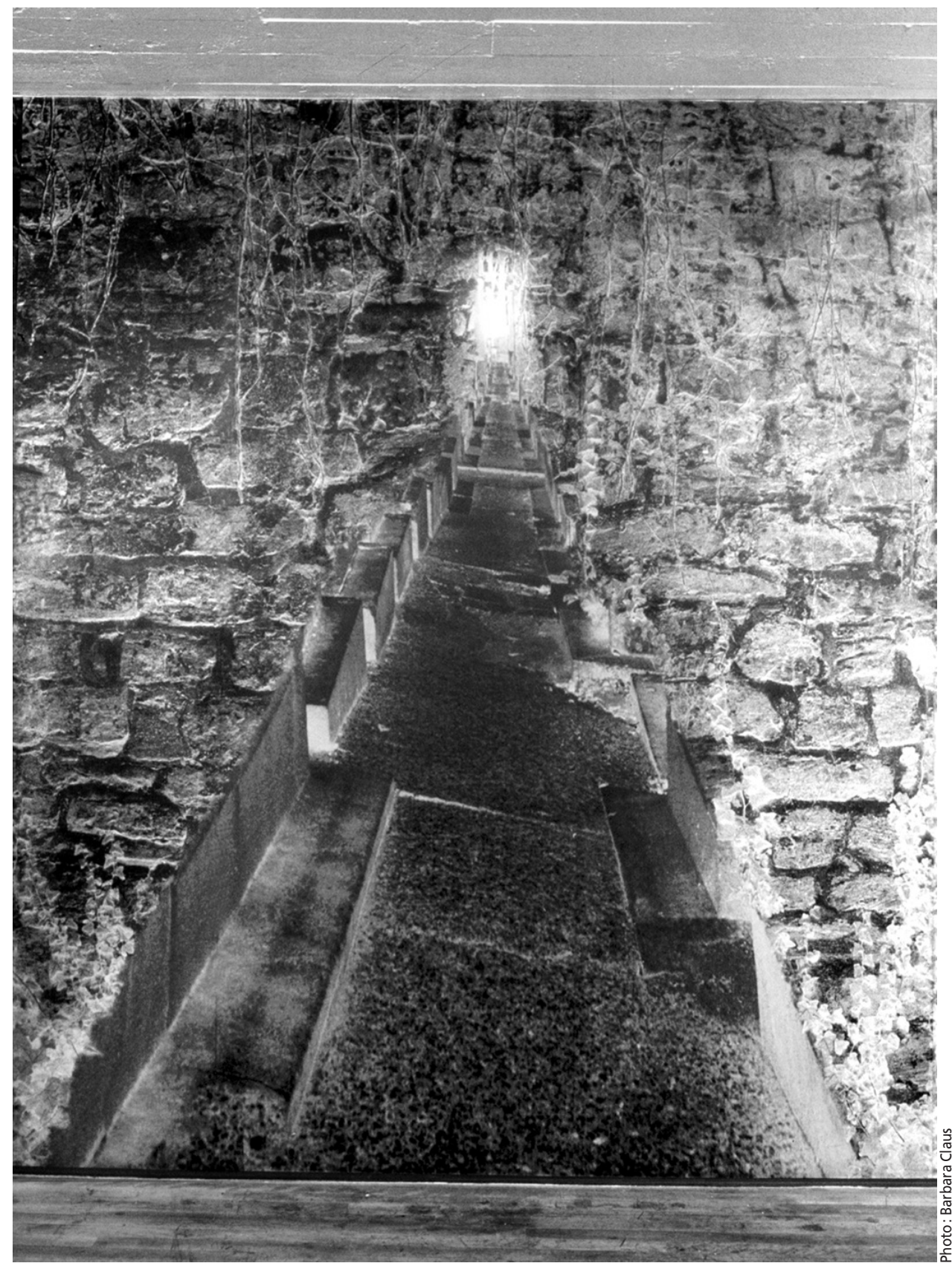

côté à cette situation, car cela peut aider les équipes à relativiser leurs difficultés. D'un autre côté, cela peut faire en sorte que les membres des équipes passent l'éponge trop facilement, trop souvent. À cet égard, une intervenante interrogée dans le cadre d'une recherche (Vachon, 1987) mentionnait qu'il était difficile, voire incongru, de se plaindre des irritants dans une atmosphère de calme comme une maison de soins palliatifs. Bref, il semble qu'en soins palliatifs on ait tendance à ne pas aborder la question des rôles et des irritants qui en découlent parce qu'il y a présence d'une règle implicite disant: quand on travaille dans ce domaine, on devrait se situer au-dessus de toutes ces tracasseries insignifiantes pour se consacrer uniquement au bien-être des patients et de leur famille, dans un climat où tout n'est que dévouement et amour. Pourtant, comme l'évoquait une intervenante (Lauzon, 1998), nier ces luttes de pouvoir, ces rivalités, n'estce pas nier en quelque sorte l'humanité des soignants pour tenter d'en faire des êtres angéliques?

Les étapes du cycle de vie d'une équipe donnent un éclairage intéressant sur une autre difficulté du travail d'équipe en soins palliatifs (Cummings, 1998). On a pu observer que, comme dans le cycle de vie familiale, il y a certaines étapes dans la vie d'une équipe. Il semble qu'il y ait une première étape qu'on pourrait intituler «lune de miel», où l'on observe un taux élevé d'enthousiasme, de fébrilité et d'idéalisme, un peu comme au début de la vie d'un couple. Durant cette période, on nie les différences. La deuxième étape, qu'on pourrait nommer celle de la différenciation, entraîne bien souvent des crises et des heurts à mesure que l'idéalisation tombe. Au cours de la troisième étape, celle de la maturation, l'équipe a réussi à trouver un certain équilibre entre l'idéal de départ et la réalité, et elle réussit à fonctionner malgré les divergences et les crises. En soins palliatifs, on peut faire l'hypothèse, en se référant à certains écrits ayant retracé l'histoire même du mouvement, qu'on a eu tendance à vouloir se maintenir à tout prix dans la première étape, celle de l'idéalisation. On considérait important de maintenir une certaine fusion afin de continuer à trouver l'énergie pour se battre avec les gens de l'extérieur pour la survie du mouvement. La citation qui suit explique de manière condensée l'importance accordée par les intervenants au fait de s'unir pour faire face à l'adversité: "Même lieu, même cause, mêmes combats, autant de raisons pour être unis contre vents et marées » (Salamagne, 1995, p. 26).

Le respect de la confidentialité peut également entraîner certaines difficultés 
dans le travail d'équipe en soins palliatifs. Lauzon (1998) a soulevé un certain nombre de questions qui mettent en relief les difficultés que peuvent provoquer au sein d'une vie d'équipe interdisciplinaire les confidences faites aux intervenants. Nous les reprenons ici de manière condensée. L'intervenant doit-il taire la confidence reçue au nom du principe de confidentialité ou le travail d'équipe exige-t-il le partage de toutes les informations susceptibles d'influencer la qualité des interventions? Est-ce qu'on doit, en tant qu'intervenant, taire certaines informations qui nous ont été confiées parce que celles-ci ne sont pas nécessaires à la compréhension de la situation ou choisira-t-on de révéler ces informations afin de conserver un certain pouvoir? Comme intervenant impliqué dans une équipe de soins palliatifs, allons-nous vouloir tout savoir parce que nous sommes curieux, voire un peu voyeurs, ou parce que nous avons besoin de toutes ces informations pour faire notre travail? Ces quelques questions laissent entrevoir qu'il n'est pas facile de trouver un équilibre entre se taire et partager, entre confidentialité et interdisciplinarité.

Enfin, une dernière difficulté réside dans la diminution du temps de séjour des patients. Avec le virage ambulatoire, on a pu constater une diminution du temps d'hospitalisation des patients dans les unités de soins palliatifs. C'est d'ailleurs un aspect qui a été documenté par la recherche (Daneault et al., 2002). Quel impact cette diminution du temps de séjour peut-elle avoir sur le travail en équipe? Il semble que, dans un tel contexte, les équipes n'aient pas toujours le temps d'arriver à une certaine cohésion, de partager les responsabilités, de coordonner l'action comme l'exige la pratique de l'interdisciplinarité. Il semble également, selon nos informateurs clés, qu'il soit difficile d'absorber les conséquences d'un tel changement dans une culture comme celle des soins palliatifs, qui valorise l'aspect relationnel.

\section{DIFFICULTÉS RENCONTRÉES DANS LES HÔPITAUX SANS UNITÉ DE SOINS PALLIATIFS ET DANS LES CLSC}

Les informations qui suivent proviennent principalement de nos informateurs clés ainsi que d'une étude menée auprès d'infirmières par Fillion et ses collaborateurs en 2003. Cette étude portait sur les stresseurs liés à la pratique infirmière en soins palliatifs. Ici, nous ferons donc entendre plus spécifiquement la voix des infirmières. En prenant connaissance des difficultés rencontrées dans le travail d'équipe en soins palliatifs dans les hôpitaux où il n'y a pas d'unité de soins palliatifs, ainsi que dans les CLSC, on constate qu'il y a changement de registre comparativement aux difficultés vécues dans le travail d'équipe dans les unités et dans les maisons de soins palliatifs. Les infirmières travaillent parfois avec des médecins non formés à la pratique des soins palliatifs et qui sont plutôt formés à guérir. Il semble qu'il soit difficile de s'ajuster à une organisation de travail qui prodigue à la fois des soins palliatifs et des soins curatifs. Les intervenants déplorent également qu'il n'y ait pas de mécanisme formel de communication entre eux. Bien souvent il n'y a pas de réunion d'équipe multidisciplinaire. Il y a des lacunes au plan de la communication entre les diverses unités impliquées et les différents quarts de travail. Il semble que la tendance soit de travailler chacun de son côté. Selon plusieurs, cela entraîne des interventions fort discutables. On relève également le manque de coordination entre les établissements (entre les hôpitaux et les CLSC), le manque de participation aux décisions, une absence de liens et de temps pour les échanges entres pairs. L'isolement que vivent les infirmières à domicile, le manque de latitude décisionnelle - il y a beaucoup de responsabilités et peu de pouvoir - et le manque d'accessibilité des médecins de famille pour ce qui est des suivis à domicile sont d'autres facteurs qui peuvent contribuer à créer des difficultés dans la vie d'équipe. Les inégalités de pouvoir entre les professions suscitent également de l'insatisfaction. Les infirmières mentionnent que les médecins ont plus de pouvoir décisionnel qu'elles dans l'hôpital. De plus, elles ont parfois l'impression que leurs compétences spécialisées en soins palliatifs ne sont pas reconnues par certains. Comme l'indiquaient déjà les données provenant des unités et des maisons de soins palliatifs, l'ambiguité et le chevauchement des rôles sont également des sources de difficultés pour le travail d'équipe dans les hôpitaux sans unité de soins palliatifs et dans les CLSC.

\section{CARACTÉRISTIQUES DES ÉQUIPES DITES VIVANTES}

Certains éléments caractérisent les équipes qui remplissent leur rôle de manière fonctionnelle (Arcand et Brissette, 1998). Dans ces équipes, on a tendance à reconnaître les problèmes sans les minimiser ni les exagérer. Les conflits sont perçus comme étant inévitables. On ne les cherche pas mais on considère qu'il est normal que des conflits émergent. Paradoxalement, il semble qu'en adoptant une telle posture, il y ait davantage de chances que l'équipe ait un fonctionnement pouvant répondre aux besoins de ses différents membres. Les chances de réussite seraient plus élevées en admettant au point de départ que la vie en équipe sera difficile et complexe. Les règles mises en place favorisent l'ouverture et l'expression des idées, des divergences et des émotions. D'ailleurs, les règles et les rôles ont tendance à être explicites et souples. Il importe ici de rappeler comment les règles implicites peuvent constituer une source d'aliénation, car il est beaucoup plus difficile de remettre en question ce qui se passe dans l'implicite. De plus, on observe que dans ce type d'équipe il y a une canalisation de l'expression des émotions et des conflits au profit du groupe. Comme le relevait Nadeau (2001), la fragilité est non seulement permise pour chacun des membres, mais aussi pour l'équipe en tant qu'entité. Dans ces mêmes équipes, on a tendance à préférer des stratégies de résolution de problèmes. Précisons ici que l'équipe ne cherche pas à entrer trop rapidement dans un mode de résolution de problème de la même façon qu'elle ne cherchera pas à prolonger indûment son autocritique. Il existe également une certaine compassion entre les membres, et il y a valorisation de toutes les tâches, qu'elles soient instrumentales ou affectives. L'équipe observe son propre fonctionnement et effectue régulièrement un examen de ce qui s'est passé. L'interdisciplinarité est également priorisée.

\section{CARACTÉRISTIQUES \\ DES ÉQUIPES DITES MORTIFÈRES}

Dans ces équipes, on observe une fermeture au changement, à ce qui vient de l'extérieur, à ce qui est perçu comme un élément menaçant. Certaines règles sont mises en place pour bloquer l'expression des idées, des divergences et des émotions. Les désaccords et les problèmes sont donc réprimés. Les différents membres ne se sentent pas soutenus. Dans ces équipes, il importe de projeter une image d'harmonie. On craint d'ailleurs bien souvent que le fait d'aborder les problèmes provoque une explosion dévastatrice. Le mythe de l'équipe parfaite et sans difficultés peut prendre de telles proportions qu'il peut paradoxalement aboutir à une dislocation de celle-ci. La présence de la peur et de la méfiance est manifeste. La crainte du changement, des gains associés au leadership dans certains sous-groupes, et la présence de quelqu'un détenant trop de pouvoir et faisant peur aux autres peuvent aussi contribuer à l'immobilisme du groupe. Plusieurs discussions se déroulent dans les corridors. Dans ces équipes, on croit d'ailleurs bien souvent que les réunions ne servent à rien et que l'essentiel est de discuter ailleurs. Il y a bien souvent présence de clans opposés et rivaux possédant chacun son chef. Dans ce type de fonctionnement, on retrouve des vedettes, c'est-àdire des personnages mythiques et sacrés 
qui sont à la fois admirés, enviés, mais aussi secrètement critiqués et craints. Comme le soulignaient Arcand et Brissette (1998), la rumination et l'entretien des conflits accaparent beaucoup d'énergie; on voit donc la productivité de ces équipes baisser. Au sein d'une équipe dite mortifère, l'intervenant dépensera beaucoup d'énergie pour agir dans une direction qui l'éloigne des motifs l'ayant poussé à s'investir dans le domaine des soins palliatifs. Le désir d'aider, de réparer, d'être utile en vient même à passer au second plan.

\section{REPÈRES POUR L'AMÉNAGEMENT DES DIFFICULTÉS}

Proposer des analogies entre le fonctionnement d'une famille et celui d'une équipe (Arcand et Brissette, 1998) peut contribuer à améliorer la vie d'équipe. Cependant, il est important de reconnaître qu'une équipe n'est pas une famille. Une équipe ne devrait pas répondre aux mêmes besoins qu'une famille. L'équipe ne doit pas être perçue comme un lieu privilégié pour recevoir difficulté du travail d'équipe, en se centrant sur la tâche et en faisant preuve d'humour (Fortin et Méthot, 2004). Certains écrits récents portant sur la résilience ont souligné comment l'humour pouvait aider à composer avec l'adversité. L'humour nous «décoince» tout en créant un soulagement émotionnel. Il permet de prendre distance et hauteur par rapport à ses problèmes (Vanistendael, 2001).

Il importe également de développer son propre système de soutien formel et informel, de distinguer ce qui appartient à soi, à l'équipe, à la structure. Si on se retrouve au sein d'une équipe ayant des caractéristiques dysfonctionnelles, il faut reconnaître l'importance de se protéger. Dans de tels contextes, il faut bien évaluer s'il n'est pas trop risqué de se montrer tels que nous sommes avec nos émotions et nos limites. Ce sont des contextes où il est probablement plus avantageux d'adopter des stratégies de cloisonnement (Arcand et Brissette, 1998). De plus, il importe d'éviter la fusion et l'absolu. Les relations de type

\section{NOUS AVONS TOUS ÉTÉ FORMÉS}

\section{DANS UNE LOGIQUE DE PROFESSIONNALISATION}

\section{ET CETTE LOGIQUE EST EN BONNE PARTIE CENTRÉE}

\section{SUR LA DÉFENSE DES TERRITOIRES PROFESSIONNELS.}

de l'affection. On peut gaspiller beaucoup d'énergie à tenter de transformer une équipe de travail en une famille ouverte. Il ne faut pas perdre de vue qu'en équipe nous devons travailler avec des personnes avec qui nous n'avons pas nécessairement d'affinités, que nous n'avons pas nécessairement choisies comme partenaires. Il semble qu'il soit beaucoup plus profitable d'accepter la réalité de l'organisation telle qu'elle est, de se centrer sur la tâche et de collaborer à son exécution, plutôt que d'essayer de transformer l'équipe en famille idéale (Fortin, 1997). À cet égard, Jacques Voyer (Fondation québécoise du cancer, 1991) disait dans une conférence: «On a eu plus que notre part de problèmes avec des gens qui avaient fait de leur implication en soins palliatifs leur investissement majeur sur le plan affectif et qui avaient décidé que le travail en soins palliatifs était ce qui leur donnerait le plus de satisfaction dans leur vie. » L'ensemble de ces propos révèle à quel point il importe de faire le deuil de l'équipe parfaite. Dans une telle posture, il ne s'agit donc pas de partir à la conquête de l'équipe idéale en multipliant les expériences ou en glorifiant tel ou tel établissement. Il s'agit plutôt de reconnaître la complexité et la fusionnel empêchent la libre expression des divergences de points de vue, car la prétendue amitié qui relie les personnes interdit l'affront direct, alors que des tensions émergent fatalement d'un travail commun. Il faut se rappeler également qu'on ne peut pas changer les autres, mais qu'on peut modifier sa propre attitude. Faire l'ermite peut parfois s'avérer salutaire. C'est bien d'être en équipe, mais c'est aussi très bien d'être seul. Donner du soutien et de l'encouragement à ses pairs et en accepter en retour constitue également une stratégie pouvant bénéficier à la vie d'équipe.

Il peut aussi se révéler aidant de reconnaître la critique constructive de celle qui est destructrice. Adopter une position critique peut aider à valider nos perceptions sans toutefois nous amener à nous enfoncer dans les ruminations. Il ne s'agit pas de sombrer ensemble mais plutôt de nous aider à nous relever. Des formations portant sur la pratique de l'interdisciplinarité de même que des retours réguliers sur le fonctionnement de l'équipe devraient permettre de composer avec certaines difficultés. Le développement de projets communs peut également permettre au facteur compétitif d'intervenir, mais dans un sens constructif.

\section{POUR CONCLURE}

Dans le projet des soins palliatifs, le travail en équipe s'est présenté comme une nécessité. Il présente cependant des caractéristiques et des difficultés qui lui sont propres, compte tenu de la culture et de l'histoire mêmes des soins palliatifs. Bien qu'il soit tentant de nier ou d'amoindrir les difficultés rencontrées, il importe de prendre en compte toute la complexité du «travailler ensemble en soins palliatifs» si l'on veut éviter que de profondes scissions s'installent dans les équipes et si l'on veut demeurer cohérents avec la philosophie même des soins palliatifs.

\section{Bibliographie}

ARCAND, M. et L. BRISSETTE (1998). Échec au burn out. Guide à l'intention des intervenants sociaux, Montréal, Chenelière/ McGraw-Hill, 140 p.

BARNES, K. (2001). «Staff stress in the children's hospice: Causes, effects and coping strategies ", International Journal of Palliative Nursing, vol. 7, n 5, p. 248-254.

BOURGEAULT, G. (1999). Éloge de l'incertitude, Montréal, Bellarmin, 178 p.

CUMMINGS, I. (1998). "The interdisciplinary team », dans D. Doyle, G. W. C. Hanks, N. Macdonald (dir.), Oxford textbook of palliative medicine, $2^{\mathrm{e}}$ édition, Oxford, Oxford University Press, p. 19-30.

D'AMOUR, D., C. SICOTTE et R. LÉVY (1999). «L'action collective au sein d'équipes interprofessionnelles dans les services de santé», Sciences Sociales et Santé, vol. 17, $n^{\circ} 3$, p. 67-92.

DANEAULT, S., D. DION, E. HUDON, S. MONGEAU, P. PAILLÉ, L. YELLE et V. LUSSIER (2002). Les soins palliatifs, victimes de leur succès? Résultats d'une étude quantitative et qualitative, communication présentée au $\mathrm{II}^{\mathrm{e}}$ Congrès du Réseau de recherche de l'Association européenne de soins palliatifs, Lyon, France.

DUSSAULT, G. (1986). «La collaboration interprofessionnelle: une utopie? », Artère, vol. $4, \mathrm{n}^{\circ} 3$, p. 17-18.

FILLION, L., L. SAINT-LAURENT et N. ROUSSEAU (2003). "Les stresseurs liés à la pratique infirmière en soins palliatifs: les points de vue des infirmières ", Les cahiers de soins palliatifs, vol. 4, n ${ }^{\circ} 1$, p. 5-40.

FONDATION QUÉBÉCOISE DU CANCER (1991). Le cancer et les intervenants, conférence filmée de Jacques Voyer, Québec.

FORTIN, B. et L. MÉTHOT (2004). «S'adapter avec humour au travail interdisciplinaire: pistes de réflexion", Revue québécoise de psychologie, vol. 25, $\mathrm{n}^{\circ} 1$, p. 99-118.

FORTIN, B. (1997). "Chapitre 8: Se situer face au groupe de travail», dans B. Fortin, Intervenir en santé mentale, Montréal, Fides, p. $82-89$. 
HÉBERT, R. (1997). «Équipe multi et interdisciplinaire », dans M. Arcand et R. Hébert (dir.). Précis pratique de gériatrie, Saint-Hyacinthe et Paris, Edisem-Maloine, p. 821-830.

HÉBERT, R. (1990). «Interdisciplinarité en gérontologie », Actes du IV congrès international francophone de gérontologie, Montréal, Edisem-Maloine, p. 55-58.

LAMAU, M.-C. (dir.) (1994). Manuel des soins palliatifs, Privat, Toulouse, $560 \mathrm{p}$.

LASSAUNIÈRE, J. M. et B. PLAGÈS (1995). "Modèles organisationnels à l'hôpital. L'interdisciplinarité», Bulletin de la Fédération Jusqu'à la Mort Accompagner la Vie, $\mathrm{n}^{\circ}$ 40, p. 35-38

LAUZON, J. (1998). «Entre le mythe et la réalité. Les soins palliatifs », Frontières, Numéro spécial $10^{\mathrm{e}}$ anniversaire, p. 43-45.

LÉVESQUE, J. (1992). «L'utilisation positive des conflits et la médiation", Intervention, $\mathrm{n}^{\circ}$ 91, p. 5-12.

MUSUMECI, J. S. (2001). The Reported Experience of a Hospice Interdisciplinary Team, thèse de doctorat, School of Education, New York University, 277 p.

NADEAU, G. (2001). «Les soins palliatifs: une option en faveur de la vie», Les cahiers de soins palliatifs, vol. 2, n ${ }^{\circ}$ 2, p. 3-29.

SALAMAGNE, M. (1995). «Vivre au pluriel dans une unité des soins palliatifs. Parole du Médecin responsable», Bulletin de la Fédération Jusqu'à la Mort Accompagner la Vie, $n^{\circ} 40$, p. 26-28.

SAUNDERS, C. (dir.) (1990). Hospice and Palliative Care. An Interdisciplinary Approach, Londres, Edward Arnold, 120 p.

SAVARD, D. (1988). La présence des bénévoles auprès des personnes confrontées à la mort, communication présentée au Colloque des soins palliatifs, Hôpital Notre-Dame, Montréal.

VACHON, M. L. (1987). «Team stress in palliative/hospice care », Hospice Journal, vol. 3, $\mathrm{n}^{\text {os }} 1-2$, p. 75-103.

VANISTENDAEL, S. (2001). «Humour et résilience: le sourire qui fait vivre», dans Fondation pour l'enfance. La résilience: le réalisme de l'espérance, Saint-Agne, Érès, p. 161-198.

\section{Note}

1. La présente contribution reprend les propos tenus lors de présentations faites dans différents colloques et activités en soins palliatifs entre 2003 et 2005.

\section{UNE NOUVELLE RESSOURCE POUR LA FORMATION DES BÉNÉVOLES EN SOINS PALLIATIFS}

Depuis les débuts du mouvement des soins palliatifs, de nombreux bénévoles ont contribué à son essor et, encore aujourd'hui, les bénévoles jouent un rôle de toute première importance dans l'évolution de ce mouvement. À travers le Québec, plusieurs équipes associées à divers milieux de soins recrutent des bénévoles et les préparent à accompagner des personnes en fin de vie ainsi que leurs proches. Un nouvel outil de formation est désormais disponible pour ces équipes, et ce, tout à fait gratuitement. Il s'agit du Programme de formation destiné à des bénévoles en soins palliatifs. La diffusion de ce programme vise à soutenir, dans le contexte de la formation de leurs bénévoles, les organisations francophones offrant des soins palliatifs. Son élaboration et sa diffusion ont été rendues possibles grâce au travail du Comité de formation du Réseau de soins palliatifs du Québec* avec l'appui et le soutien financier de la Fondation PalliAmi associée à l'Unité de soins palliatifs (USP) de l'Hôpital Notre-Dame du Centre hospitalier de l'Université de Montréal. Conçu d'abord pour l'USP de l'Hôpital Notre-Dame, ce programme propose un contenu théorique de base accompagné de stratégies de formation dont toute organisation peut largement s'inspirer. Il a été élaboré de manière à pouvoir être facilement adapté par chacune des organisations qui voudrait y avoir recours pour la formation de ses bénévoles. D'une durée suggérée d'au moins douze heures, ce programme est basé en grande partie sur la reconnaissance et la mise en valeur des forces et des aptitudes des bénévoles, tout en leur permettant d'approfondir leur compréhension du rôle de bénévole auprès de personnes en fin de vie et de leurs proches. Un autre point d'ancrage de ce programme de formation est l'échange (d'idées, de sentiments, d'expériences...) et l'écoute qui encourage et facilite cet échange.

\section{Pour plus d'informations: <www.palliami.org >.}

\author{
* Claudette Foucault, coordonnatrice du comité, \\ Manon Champagne, coordonnatrice du projet pour la Fondation PalliAmi, \\ Danielle J. Drouin, Suzanne Mongeau et Michèle Viau-Chagnon.
}

